\title{
Management of Storage Diseases of Onion by Using Different Botanicals
}

\author{
A.S. Futane ${ }^{1 *}$, B.P. Dandnaik ${ }^{2}$, P.P. Jadhav ${ }^{1}$ and S.S. Salunkhe ${ }^{1}$ \\ ${ }^{1}$ Department of plant pathology, College Of Agriculture, Latur, India \\ ${ }^{2}$ Department of plant pathology, college of Agriculture, Osmanabad, VNMKV, \\ Parbhani, India \\ *Corresponding author
}

\section{A B S T R A C T}

\begin{tabular}{|l|}
\hline Ke y w o r d s \\
Storage diseases, \\
$\begin{array}{l}\text { Onion, Different } \\
\text { botanicals, Allium }\end{array}$ \\
\hline Article Info \\
\hline $\begin{array}{l}\text { Accepted: } \\
\text { 28 February } 2018 \\
\text { Available Online: } \\
\text { 10 March 2018 }\end{array}$ \\
\hline
\end{tabular}

\section{Introduction}

Onion (Allium cepa L.), is a member of class Liliaceae and family Alliaceae, widely distributed and grown in tropical, sub-tropical and temperate climatic zones (Fritsch and Friesen, 2002). It is biennial crop (Bohanec et al., 2003), and in India it is grown twice (Rabi and Kharif) in a year (NHRDF, 2009). The name "wild onion" is applied to a number of Allium species but A. cepa is exclusively known from cultivation and its wild original form is not known.

Onion rightly called as "Queen of kitchen" is one of the oldest known and an important vegetable crop grown in India Onion is supposed to have its origin in the Middle East Asian Countries and introduced in India from Palestine. It belongs to family Alliaceae, and genus Allium with about 300 species. As vegetable and spice it is used both as tender and mature bulb. In bulb group vegetables the most important crop is onion. The bulb composed of concentric, fleshy, in large leaf bases or scales. Onion contains phenolics and flavonoids that have potential antiinflammatory, anticolesterols, anticancer and antioxidant properties. Onion is grown in three season i.e. Kharif, Rabi, and summer. In Kharif season, during May-June seeds are sown and transplanted during July-Aug months and onion become ready for harvesting during Oct-Nov month. 
The post - harvest losses, viz. sprouting, rotting, and physiological loss in weight pose a great problem. It is reported that annual storage losses were over 40 per cent (Bhagachandani et al., 1980) and between 4060 per cent (Maini et al., 1984) in India.

The onion produce is available in market during October - November (20 per cent) as kharif crop, January- February (20 per cent) as late Kharif crop and April-May (60 per cent) as rabi crop. The rabi crop produce having more ability in storage and used for domestic, export and seed bulbs purposes form June to November. This is the critical period in whole country, where there is no fresh harvest onion and hence, storage become paramount importance for steady supply. Nearly two million tones needs to be stored during this period (Tripathi and Lawande, 2003).

Losses of onion during storage are considerable mainly due to sprouting and contamination by several microorganisms. Nearly $40 \%$ of the production is lost during post harvest handling and sprouting. Microbial spoilage alone contributes approximately 15$20 \%$ of the total loss (Pantastico and Bantista, 1976; Bhagchandani et al., 1980). Earlier, (Quadri et al., 1982) stated that the spoilage caused by Aspergillus niger was as high as $80 \%$.

\section{Materials and Methods}

\section{Experimental site}

All the experiments (In vitro) were conducted at the Department of Plant Pathology, College of Agriculture, Latur.

\section{Collection of disease samples}

Onion bulbs showing symptoms of rot, black, brown and discolouration were randomly collected in the bags from the various markets and fields in the Osmanabad and Latur district. These collected bulbs were brought to the Plant Pathology, Laboratory, College of Agriculture, Latur and subjected for further studies.

\section{Culture media}

Potato dextrose agar (PDA), the common laboratory culture medium was used as basal medium for isolation, purification, multification and maintenance of the pure culture of diseases.

\section{Chemicals}

Standard chemicals, reagents, fungicides, culture media etc. required for the experimentation were obtained from the department of Plant Pathology, college of Agriculture, Latur.

\section{Glass-wares}

The common glass-wares (Borosil and corning make) viz., Petri dishes, test tubes, conical flasks, volumetric flasks, measuring cylinder, glass rods, beakers, funnel, pipettes etc. were obtained from the Department of Plant Pathology, College of Agriculture, Latur.

\section{Equipments}

The laboratory equipments viz., Autoclave, Hot air oven, Laminar-airflow Cabinet, BOD incubator, Refrigerator, Binocular Research Microscope, Electronic balance, $\mathrm{pH}$ meter, Mixer-cum-grinder etc. available at the Department of Plant Pathology, College of Agriculture, Latur were utilized, as and when required.

\section{Miscellaneous}

Inoculation needle, forceps, blotter papers, paper bags, polythene bags, spirit lamp, 
mercuric chloride, labels, scales, etc. available at the Department of Plant Pathology were used.

\section{Plant extracts/botanicals}

Plant species reported with potential antifungal and therapeutic properties (Alice, 1984) against fungal pathogens and available locally were collected from the farms of Oilseeds Research Station, College of Agriculture, Latur and adjoining fields. Following locally available 9 plant species/botanicals were used for in vitro studies.

\section{In vitro evaluation of botanicals (Plant extracts)}

Plant leaf extracts of nine botanicals viz., Tulsi, Garlic, Ginger, Ashoka, Dhatura, Lantena camera, Jakhamjudi, Karanj, and Neem oil were evaluated against Aspergillus niger and Fusarium oxysporum f.sp.cepae. Leaf extracts were prepared by grinding with mixer cum grinder the $100 \mathrm{~g}$ washed leaves of each plant species in $100 \mathrm{ml}$ distilled water and filtered through double layered muslin cloth. The filtrates obtained were further filtered through Whatman No. 1 filter paper using funnel and volumetric flasks $(100 \mathrm{ml}$ cap.). The final clear extracts obtained formed the standard leaf extracts of $100 \%$ concentration, which were evaluated by applying poisoned food technique.

For the purpose, PDA was used as basal culture medium. An appropriate quantity of each leaf extract (100\%) was separately mixed thoroughly in PDA medium in conical flasks (250 $\mathrm{ml}$ cap.) to obtain desired concentrations i.e. $10 \%, 15 \%$ and $20 \%$ and autoclaved at 15 lbs/inch ${ }^{2}$ pressure for 15 to 20 minutes. Sterilized and cooled PDA amended with leaf extracts was then poured (15 to $20 \mathrm{ml} /$ plate) into sterile glass Petri plates (90 mm dia.) and allowed the medium to solidify at room temperature. Each plant leaf extract and its respective concentrations were replicated thrice. The plates containing PDA without any extract were maintained as control. Upon solidification of PDA, all the plates were aseptically inoculated by placing in the centre a $5 \mathrm{~mm}$ mycelial disc obtained from a week old culture of $A$. niger grown on agar plates. Plates containing plain PDA and inoculated with test fungus served as untreated control. All these plates were then incubated at $27 \pm 1^{0}$ $\mathrm{C}$ temperature for a week or till the untreated control plates were fully covered with mycelial growth of the test fungus.

\section{Experimental details}

Design: C.R.D.

Replication: Three

Treatment: Ten

T1: Ginger (Zingiber Officinale)

T2: Tulsi (Osmium sanctum)

T3: Garlic (Allium Sativum)

T4: Ashok (Polyalthia longifolia)

T5: Dhatura (Datura metal)

T6: Ghaneri (Lantana camera)

T7: Jakhamjudi (Tridax procumbens)

T8: Karanj (Pongamia pinnata)

T9: Neem oil (Azadirachta indica)

T10: Control

Observations on radial mycelial growth/ colony diameter of the test fungus were recorded treatment wise at 24 hours intervals and continued till mycelial growth of the test fungus was fully covered in the untreated control plates. Per cent inhibitions of mycelia growth over untreated control were calculated by applying the formula given by Vincent (1947).

Colony growth in control plate Colony growth in poisoned plate

Inhibition $(\%)=$--------------------------- x 100

Colony growth in control plate 


\section{Statistical analysis}

The data obtained in all the experiments (in vitro) was statistically analyzed. The percentage values were transformed into Arcsine values. The standard error (SE) and critical difference (C.D.) at level $\mathrm{P}=0.01 \%$ were worked out and results obtained were compared statistically.

\section{Results and Discussion}

In vitro efficacy of plant extracts against $A$. niger

A total of nine botanicals viz., tulsi (Osimum sanctum), garlic (Allium sativum), ginger (zingiber officinale), ashok (Polyalthia longifolia), datura (Datura metal), ghaneri (Lantana camera), Jakhamjudi (Tridax procumbens), karanj (Pongamia pinnata), neem oil (Azadirachta indica) were evaluated (@10,15 and $20 \%$ each) in vitro against $A$. niger applying Poisoned Food Technique (Nene and Thapliyal, 1993) and using PDA as a basal medium.

Effect of these botanicals / phytoextracts on radial mycelial growth and inhibition of the test pathogen were recorded. All the treatments were replicated thrice and a suitable untreated control (without plant extract) was also maintained.

\section{Radial mycelial growth}

Result revealed that all the botanicals / plant extracts tested exhibited a varied range of radial mycelial growth of the test pathogen and depending upon their concentrations used and it was decreased with increase in concentrations of the botanicals tested. At 10 per cent, radial mycelial growth of the test pathogen ranged from $47.85 \mathrm{~mm}$ (A. sativum) to $83.04 \mathrm{~mm}$ (D. metal). However, it was maximum with D. metal $(83.04 \mathrm{~mm})$. This was followed by $P$. longifolia $(81.42 \mathrm{~mm}), \quad Z$. officinale $(80.19 \mathrm{~mm})$, P. pinnata $(78.21 \mathrm{~mm})$, T. procumbens $(78.09 \mathrm{~mm}), \quad$ L. camera $(77.14 \mathrm{~mm}), A$. indica $(69.99 \mathrm{~mm})$ and $O$. sanctum $(57.96 \mathrm{~mm})$. Significantly least radial mycelial growth was recorded with A. sativum $(47.85 \mathrm{~mm})$ over untreated control $(90.00 \mathrm{~mm})$.

At 15 per cent, radial mycelial growth of the test pathogen ranged from $42.67 \mathrm{~mm}$ (A. sativum) to $80.15 \mathrm{~mm}$ (D. metal). However, it was maximum with $D$. metal $80.15 \mathrm{~mm}$. This was followed by P. longifolia $(78.30 \mathrm{~mm}), T$. procumbens $\quad(76.94 \mathrm{~mm}) . \quad Z$. officinale (76.66mm), P. pinnata $(76.50 \mathrm{~mm})$, L. camera $(74.15 \mathrm{~mm}), A$. indica $(68.22 \mathrm{~mm})$ and $O$. sanctum $(53.73 \mathrm{~mm})$. Significantly least radial mycelial growth was recorded with A. sativum $(42.67 \mathrm{~mm})$ over untreated control $(90.00 \mathrm{~mm})$.

At 20 per cent, radial mycelial growth of the test pathogen ranged from $36.32 \mathrm{~mm}$ (A. sativum) to $78.00 \mathrm{~mm}$ (D. metal). However, it was maximum with $D$. metal $(78.00 \mathrm{~mm})$. This was followed by $P$. longifolia $(76.98 \mathrm{~mm}), T$. procumbens $\quad(75.98 \mathrm{~mm}), \quad P$. pinnata $(75.14 \mathrm{~mm}), \quad$ L. camera $(73.25 \mathrm{~mm}), \quad Z$. officinale $(71.17 \mathrm{~mm})$, A. indica $(65.50 \mathrm{~mm})$ and $O$. sanctum $(49.29 \mathrm{~mm})$. Significantly least radial mycelial growth was recorded with $A$. sativum $(36.32 \mathrm{~mm})$ over untreated control $(90.00 \mathrm{~mm})$.

The mean radial mycelial growth recorded with the plant extracts tested (@10,15 and 20 $\%$ each) ranged from $42.01 \mathrm{~mm}$ (A. sativum) to $80.39 \mathrm{~mm}$ (D. metal). However, highest mean radial mycelial growth was recorded with $D$. metal $(80.39 \mathrm{~mm})$, and was followed by $P$. longifolia $(78.90 \mathrm{~mm}), \quad$ T. procumbens (77.00mm), $\quad P . \quad$ pinnata $(76.61 \mathrm{~mm}), \quad Z$. officinale $(76.00 \mathrm{~mm})$, L. camera $(74.84 \mathrm{~mm})$, $A$. indica $(67.90 \mathrm{~mm})$, and $O$. sanctum $(53.66 \mathrm{~mm})$. Significantly least radial mycelial growth was recorded with $A$. sativum $(42.01 \mathrm{~mm})$, over untreated control $(90.00 \mathrm{~mm})$. 


\section{Mycelial growth inhibition}

Result revealed that all the plant extracts tested, significantly inhibited mycelial growth of the tests fungus over untreated control $(00.00 \%)$. Further, it was found that per cent mycelial growth inhibition of the test pathogens was increased with increase in concentrations of the botanicals tested. At 10 per cent, radial mycelial growth inhibition of the test pathogen ranged from $7.73 \%$ ( $D$. metal)) to $46.83 \%$ (A. sativum). However, significantly highest mycelial growth inhibition was recorded with $A$. sativum (46.83\%). This was followed by $O$. sanctum (35.60\%), A. indica (22.23\%), L. camera $(14.28 \%)$ and least growth inhibition was recorded, $T$. procumbens, $P$. pinnata, $Z$. officinale, $P$. longifolia and D. metal. over untreated control $(00.00 \%)$.

At 15 per cent, radial mycelial growth inhibition of the test pathogen ranged from $10.94 \%$ (D. metal) to $52.58 \%$ (A. sativum). However, significantly highest mycelial growth inhibition was recorded with $A$. sativum $(52.58 \%)$. This was followed by $O$. sanctum (40.30\%), A. indica (24.20\%), L. camera (17.61\%), P. pinnata (14.99\%), Z. officinale $(14.82 \%)$, T. procumbens $(14.51 \%)$, $P$. longifolia $(13.00 \%)$ and very less growth inhibition was recorded D. metal $(10.94 \%)$ over untreated control $(00.00 \mathrm{~mm})$.

At 20 per cent, radial mycelial growth inhibition of the test pathogen ranged from $13.33 \%$ (D. metal) to $59.64 \%$ (A. sativum). However, significantly highest mycelial growth inhibition was recorded with $A$. sativum $(59.64 \%)$. This was followed by $O$. sanctum (45.23\%), A. indica (27.22\%), $Z$. officinale $(20.92 \%)$ and very less growth inhibition was recorded, L. camera $(18.61 \%)$, $P$. pinnata (16.51\%), $T$. procumbens (15.57\%), P. longifolia (14.46\%) and D. metal $(13.33 \%)$ over untreated control $(00.00 \mathrm{~mm})$.
Mean percentage mycelial growth inhibition recorded with all the botanicals tested ranged from $10.66 \%$ (D. metal) to $53.01 \%$ (A. sativum). However, A. sativum was found most fungistatic and recorded significantly highest mean mycelial growth inhibition $(53.01 \%)$. The second and third best plant extracts found were $O$. sanctum (40.37\%) and A. indica $(24.55 \%)$. The remaining all plant extracts found least effective against the test pathogen

Thus, on the basis of mean mycelial growth inhibition, the botanicals were found most antifungal against Aspergillus niger. The effectiveness of these botanicals extracts may be due to the presence of bioactive and antifungal compounds like phenolic substances, nonvolatile compounds. The presence of ajoene and alliicin in A. sativum might be the reason for inhibition of Aspergillus niger (Yoshida et al., 1987; Naganawa et al., 1996). The ineffectiveness of the other plant extracts on $A$. niger might be due to insolubility of their active compounds in water (Qasem and Abu-Blan, 1996; Amadioha, 2000).

These results of the present study are in consonance with the finding of several previous workers. Botanical / plant extracts viz., A. sativum, $Z$. officinale, D. metal, P.pinnota and $A$. indica were reported antifungal / fungistatic against Aspergillus niger, earlier by several workers (Irkin and Korukluoglu, 2007; Avasthi et al., 2010; Sonawane et al., 2012; Khan and Nasreen 2013;)

\section{In vitro efficacy of plant extracts against Fusarium oxysporum f. sp. cepae}

A total of nine botanicals viz., tulsi (Osimum sanctum), garlic (Allium sativum), ginger (zingiber officinale), ashok (Polyalthia longifolia), datura (Datura metal), ghaneri 
(Lantana camera), Jakhamjudi (Tridax procunbenx), karanj (Pongamia pinnata), neem oil (Azadirachta indica) were evaluated (@10,15 and $20 \%$ each) in vitro against Fusarium oxysporum f.sp.cepae, applying Poisoned Food Technique (Nene and Thapliyal, 1993) and using PDA as a basal medium. Effect of these botanicals / phytoextracts on radial mycelial growth and inhibition of the test pathogen were recorded. All the treatments were replicated thrice and a suitable untreated control (without plant extract) was also maintained.

\section{Radial mycelial growth}

Result revealed that all the botanicals / plant extracts tested exhibited a varied range of radial mycelial growth of the test pathogen and depending upon their concentrations used and it was decreased with increase in concentrations of the botanicals tested.

In vitro efficacy of the botanicals on mycelial growth and inhibition of Aspergillus niger

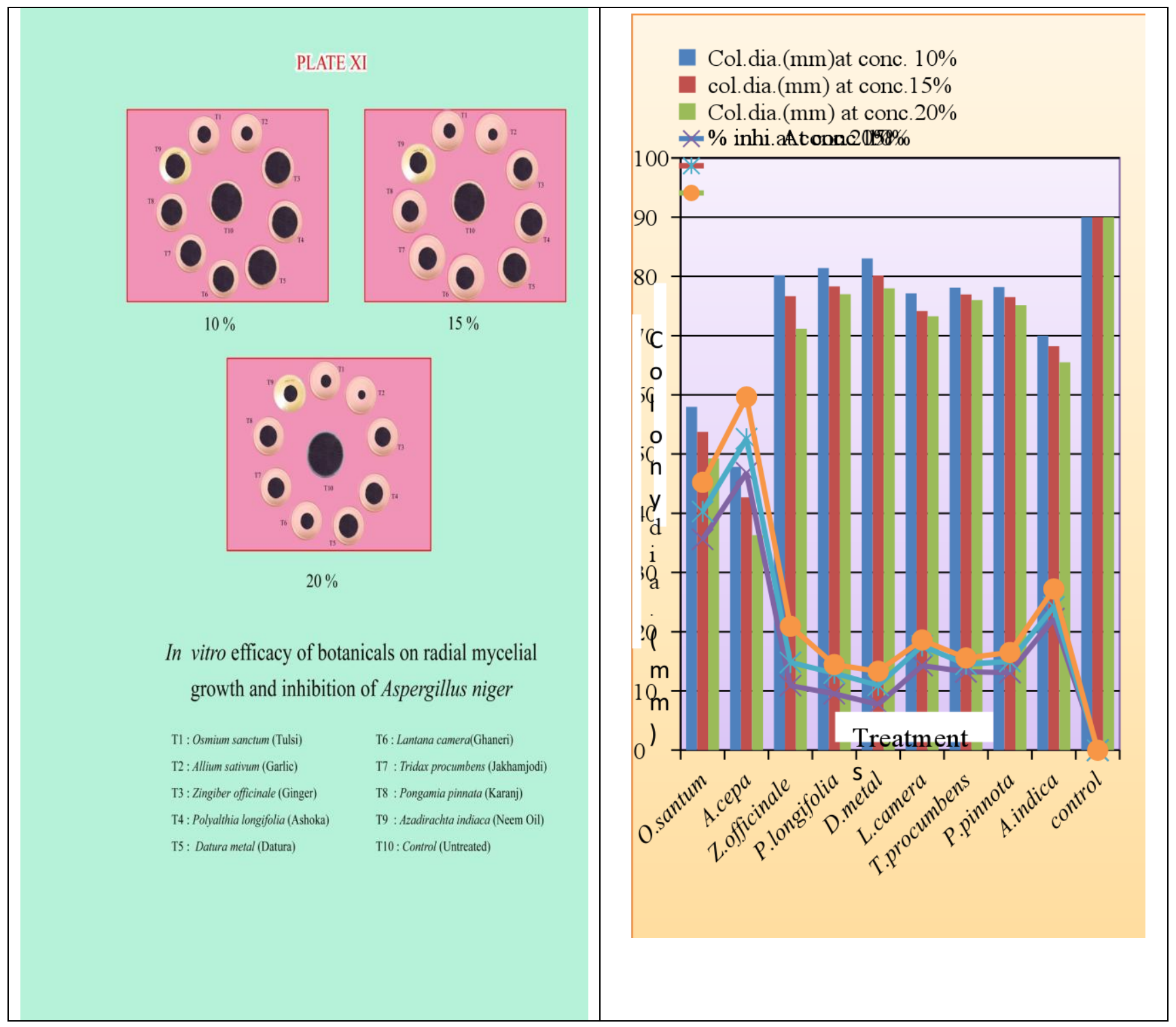




\section{In vitro efficacy of the botanicals on mycelial growth and inhibition of Fusarium oxysporum $f$. sp. cepae}

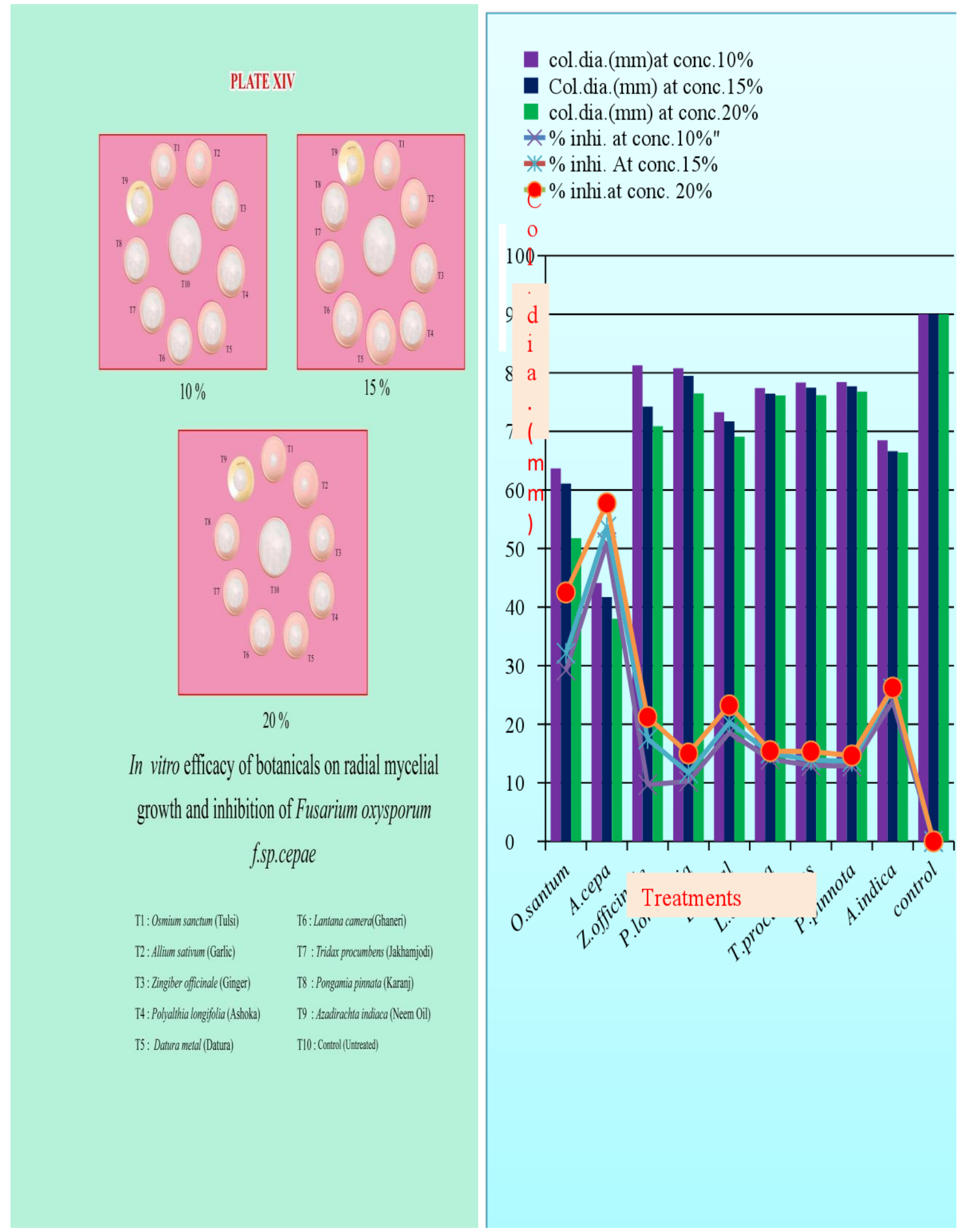


Plant extracts/botanicals

\begin{tabular}{|c|c|c|c|c|c|c|c|}
\hline $\begin{array}{l}\text { Sr. } \\
\text { No }\end{array}$ & $\begin{array}{l}\text { Local } \\
\text { name }\end{array}$ & $\begin{array}{l}\text { Scientific } \\
\text { Name }\end{array}$ & $\begin{array}{c}\text { Plant Part } \\
\text { Used }\end{array}$ & $\begin{array}{l}\text { Sr. } \\
\text { No. }\end{array}$ & $\begin{array}{l}\text { Local } \\
\text { name }\end{array}$ & $\begin{array}{l}\text { Scientific } \\
\text { Name }\end{array}$ & $\begin{array}{c}\text { Plant Part } \\
\text { Used }\end{array}$ \\
\hline 1. & Ginger & $\begin{array}{l}\text { Zingiber } \\
\text { Officinale }\end{array}$ & Rhizome & 6 & Ghaneri & $\begin{array}{l}\text { Lantana } \\
\text { camera }\end{array}$ & Leaf \\
\hline 2. & Tulsi & $\begin{array}{l}\text { Osmium } \\
\text { sanctum }\end{array}$ & Leaf & 7 & Jakhamjudi & $\begin{array}{c}\text { Tridax } \\
\text { procumbens }\end{array}$ & Leaf \\
\hline 3. & Garlic & Allium Sativum & Bulb/Petal & 8 & Karanj & $\begin{array}{l}\text { Pongamia } \\
\text { pinnata }\end{array}$ & Leaf \\
\hline 4. & Ashoka & $\begin{array}{l}\text { Polyalthia } \\
\text { longifolia }\end{array}$ & Leaf & 9 & Neem oil & $\begin{array}{c}\text { Azadirachta } \\
\text { indica }\end{array}$ & Oil \\
\hline
\end{tabular}

5. Dhatura Datura metal Leaf

In vitro effect of different plant extracts on mycelia growth and inhibition of $A$. niger

\begin{tabular}{|c|c|c|c|c|c|c|c|c|c|}
\hline \multirow[t]{2}{*}{$\begin{array}{l}\text { Tr. } \\
\text { No. }\end{array}$} & \multirow[t]{2}{*}{$\begin{array}{c}\text { Treatme } \\
\text { nts }\end{array}$} & \multicolumn{3}{|c|}{$\begin{array}{l}\text { Colony Diameter } \\
(\mathrm{mm})^{*} \text { at conc. }\end{array}$} & \multirow[t]{2}{*}{$\begin{array}{l}\text { Mean } \\
(\mathbf{m m})\end{array}$} & \multicolumn{3}{|c|}{$\%$ Inhibition* at conc. } & \multirow{2}{*}{$\begin{array}{c}\text { Mean } \\
\% \\
\text { Inhib. }\end{array}$} \\
\hline & & $10 \%$ & $15 \%$ & $20 \%$ & & $10 \%$ & $15 \%$ & $20 \%$ & \\
\hline T1 & Tulsi & 57.96 & 53.73 & 49.29 & 53.66 & $\begin{array}{c}35.6 \\
(20.86)\end{array}$ & $\begin{array}{c}40.30 \\
(23.76)\end{array}$ & $\begin{array}{c}45.23 \\
(26.89)\end{array}$ & $\begin{array}{c}40.37 \\
(23.83)^{*}\end{array}$ \\
\hline $\mathrm{T} 2$ & Garlic & 47.85 & 42.67 & 36.32 & 42.01 & $\begin{array}{c}46.83 \\
(27.94)\end{array}$ & $\begin{array}{c}52.58 \\
(31.72)\end{array}$ & $\begin{array}{c}59.64 \\
(36.62)\end{array}$ & $\begin{array}{c}53.01 \\
(32.09)\end{array}$ \\
\hline T3 & Ginger & 80.19 & 76.66 & 71.17 & 76.00 & $\begin{array}{c}10.9 \\
(6.25)\end{array}$ & $\begin{array}{l}14.82 \\
(8.48)\end{array}$ & $\begin{array}{c}20.92 \\
(12.07)\end{array}$ & $\begin{array}{l}15.54 \\
(8.93)\end{array}$ \\
\hline $\mathrm{T} 4$ & Ashok & 81.42 & 78.30 & 76.98 & 78.90 & $\begin{array}{c}9.53 \\
(5.46)\end{array}$ & $\begin{array}{c}13 \\
(7.46)\end{array}$ & $\begin{array}{l}14.46 \\
(8.31)\end{array}$ & $\begin{array}{l}12.33 \\
(7.07)\end{array}$ \\
\hline T5 & Datura & 83.04 & 80.15 & 78.00 & 80.39 & $\begin{array}{c}7.73 \\
(4.43)\end{array}$ & $\begin{array}{l}10.94 \\
(6.28)\end{array}$ & $\begin{array}{l}13.33 \\
(7.66)\end{array}$ & $\begin{array}{l}10.66 \\
(6.12)\end{array}$ \\
\hline T6 & Ghaneri & 77.14 & 74.15 & 73.25 & 74.84 & $\begin{array}{l}14.28 \\
(8.20)\end{array}$ & $\begin{array}{c}17.61 \\
(10.13)\end{array}$ & $\begin{array}{c}18.61 \\
(10.71)\end{array}$ & $\begin{array}{l}16.83 \\
(9.68)\end{array}$ \\
\hline $\mathrm{T} 7$ & $\begin{array}{c}\text { Jakhamju } \\
\text { di }\end{array}$ & 78.09 & 76.94 & 75.98 & 77.00 & $\begin{array}{l}13.23 \\
(7.59)\end{array}$ & $\begin{array}{l}14.51 \\
(8.33)\end{array}$ & $\begin{array}{l}15.57 \\
(8.99)\end{array}$ & $\begin{array}{l}14.43 \\
(8.30)\end{array}$ \\
\hline $\mathrm{T} 8$ & Karanj & 78.21 & 76.50 & 75.14 & 76.61 & $\begin{array}{l}13.10 \\
(7.51)\end{array}$ & $\begin{array}{l}14.99 \\
(8.57)\end{array}$ & $\begin{array}{l}16.51 \\
(9.86)\end{array}$ & $\begin{array}{l}14.86 \\
(8.64)\end{array}$ \\
\hline T9 & Neem oil & 69.99 & 68.22 & 65.50 & 67.90 & $\begin{array}{c}22.23 \\
(12.84)\end{array}$ & $\begin{array}{c}24.20 \\
(14)\end{array}$ & $\begin{array}{c}27.22 \\
(15.79)\end{array}$ & $\begin{array}{c}24.55 \\
(14.21)\end{array}$ \\
\hline \multirow[t]{3}{*}{ T10 } & Control & 90.00 & 90.00 & 90.00 & 90.00 & 00.00 & 00.00 & 00.00 & 00.00 \\
\hline & S.E.+- & 0.96 & 0.72 & 0.70 & - & 0.70 & 0.50 & 0.53 & - \\
\hline & $\begin{array}{c}\text { C.D. } \\
(P=0.01)\end{array}$ & 2.85 & 2.12 & 2.07 & - & 2.08 & 1.49 & 1.58 & - \\
\hline
\end{tabular}

*: Figures in parenthesis arc sin transformed value

Mean of three replications

Conc. $=$ Concentration

Inhib. $=$ Inhibition 
In vitro effect of different plant extracts on growth and inhibition of Fusarium oxysporum $f$. sp. cepae

\begin{tabular}{|c|c|c|c|c|c|c|c|c|c|}
\hline \multirow[t]{2}{*}{$\begin{array}{l}\text { Tr. } \\
\text { No. }\end{array}$} & \multirow[t]{2}{*}{ Treatments } & \multicolumn{3}{|c|}{$\begin{array}{l}\text { Colony Diameter } \\
\text { (mm)*at conc. }\end{array}$} & \multirow[t]{2}{*}{$\begin{array}{l}\text { Mean } \\
(\mathrm{mm})\end{array}$} & \multicolumn{3}{|c|}{$\%$ Inhibition at conc. } & \multirow{2}{*}{$\begin{array}{c}\text { Mean } \\
\% \\
\text { Inhib. }\end{array}$} \\
\hline & & $10 \%$ & $15 \%$ & $20 \%$ & & $10 \%$ & $15 \%$ & $20 \%$ & \\
\hline $\mathrm{T} 1$ & Tulsi & 63.70 & 61.09 & 51.75 & 61.21 & $\begin{array}{c}29.22 \\
(16.98)\end{array}$ & $\begin{array}{c}32.12 \\
(18.73)\end{array}$ & $\begin{array}{c}42.50 \\
(25.15)\end{array}$ & $\begin{array}{c}34.61 \\
(20.28)^{*}\end{array}$ \\
\hline $\mathrm{T} 2$ & Garlic & 44.10 & 41.71 & 38.02 & 41.27 & $\begin{array}{c}50.99 \\
(30.65)\end{array}$ & $\begin{array}{c}53.65 \\
(32.44)\end{array}$ & $\begin{array}{c}57.75 \\
(35.27)\end{array}$ & $\begin{array}{c}54.13 \\
(32.78)\end{array}$ \\
\hline T3 & Ginger & 81.29 & 74.24 & 70.88 & 75.47 & $\begin{array}{c}9.67 \\
(5.54)\end{array}$ & $\begin{array}{c}17.51 \\
(10.08)\end{array}$ & $\begin{array}{c}21.24 \\
(12.26)\end{array}$ & $\begin{array}{l}16.14 \\
(9.29)\end{array}$ \\
\hline $\mathrm{T} 4$ & hok & 80.77 & 79.47 & 76.49 & 55.57 & $\begin{array}{l}10.25 \\
(5.88)\end{array}$ & $\begin{array}{l}11.69 \\
(6.71)\end{array}$ & $\begin{array}{l}15.01 \\
(8.67)\end{array}$ & $\begin{array}{l}12.31 \\
(7.08)\end{array}$ \\
\hline T5 & Datura & 73.27 & 71.71 & 69.09 & 71.35 & $\begin{array}{c}18.58 \\
(10.70)\end{array}$ & $\begin{array}{c}20.32 \\
(11.72)\end{array}$ & $\begin{array}{c}23.23 \\
(13.43)\end{array}$ & $\begin{array}{c}20.71 \\
(11.95)\end{array}$ \\
\hline T6 & Ghaneri & 77.38 & 76.43 & 76.12 & 76.64 & $\begin{array}{l}14.02 \\
(8.03)\end{array}$ & $\begin{array}{l}15.07 \\
(8.70)\end{array}$ & $\begin{array}{l}15.42 \\
(8.90)\end{array}$ & $\begin{array}{l}14.83 \\
(8.54)\end{array}$ \\
\hline $\mathrm{T} 7$ & Jakhamjudi & 78.33 & 77.48 & 76.18 & 77.33 & $\begin{array}{l}12.96 \\
(7.43)\end{array}$ & $\begin{array}{c}13.91 \\
(7.98)\end{array}$ & $\begin{array}{l}15.35 \\
(8.86)\end{array}$ & $\begin{array}{l}14.07 \\
(8.09)\end{array}$ \\
\hline T8 & Karanj & 78.43 & 77.70 & 76.80 & 77.64 & $\begin{array}{r}12.85 \\
(7.36)\end{array}$ & $\begin{array}{l}13.66 \\
(7.46)\end{array}$ & $\begin{array}{l}14.66 \\
(8.42)\end{array}$ & $\begin{array}{l}13.72 \\
(7.74)\end{array}$ \\
\hline T9 & Neem oil & 68.49 & 66.60 & 66.38 & 67.75 & $\begin{array}{c}23.9 \\
(13.82)\end{array}$ & $\begin{array}{c}26 \\
(15.06)\end{array}$ & $\begin{array}{c}26.24 \\
(21.24)\end{array}$ & $\begin{array}{c}28.71 \\
(16.70)\end{array}$ \\
\hline \multirow[t]{3}{*}{$\mathrm{T} 10$} & Control & 90.00 & 90.00 & 90.00 & 90.00 & 00.00 & 00.00 & 00.00 & 00.00 \\
\hline & S.E. \pm & 0.49 & 0.54 & 0.64 & - & 0.35 & 0.37 & 0.45 & - \\
\hline & $\begin{array}{c}\text { C.D. } \\
(P=0.01)\end{array}$ & 1.45 & 1.59 & 1.90 & - & 1.04 & 1.10 & 1.35 & - \\
\hline
\end{tabular}

*: Figures in parenthesis arc sin transformed value Average of three replications

Conc. $=$ Concentration Inhib. $=$ Inhibition

At 10 per cent, radial mycelial growth of the test pathogen ranged from $44.10 \mathrm{~mm}(A$. sativum) to $81.29 \mathrm{~mm}$ (Z. officinale). However, it was maximum with $Z$. officinale $(81.29 \mathrm{~mm})$. This was followed by $P$. longifolia $(80.77 \mathrm{~mm})$, P. pinnata $(78.43 \mathrm{~mm})$, T. procumbens $(78.33 \mathrm{~mm}), \quad$ L. camera $(77.38 \mathrm{~mm})$, D. metal $(73.27 \mathrm{~mm})$, A. indica $(68.49 \mathrm{~mm})$ and $O$. sanctum $(63.70 \mathrm{~mm})$. Significantly least radial mycelial growth was recorded with A. sativum $(44.10 \mathrm{~mm})$ over untreated control $(90.00 \mathrm{~mm})$. At 15 per cent, radial mycelial growth of the test pathogen ranged from $41.71 \mathrm{~mm}$ (A. sativum) to
$79.47 \mathrm{~mm}$ (P. longifolia). However, it was maximum with $P$. longifolia $(79.47 \mathrm{~mm})$. This was followed by $P$. pinnata $(77.70 \mathrm{~mm}), T$. procumbens $(77.48 \mathrm{~mm}), \quad$ L. camera (76.43mm), Z. officinale $(74.24 \mathrm{~mm})$, D. metal $(71.71 \mathrm{~mm}), A$. indica $(66.60 \mathrm{~mm})$ and $O$. sanctum $(61.09 \mathrm{~mm})$. Significantly least radial mycelial growth was recorded with A. sativum $(41.71 \mathrm{~mm})$ over untreated control $(90.00 \mathrm{~mm})$.

At 20 per cent, radial mycelial growth of the test pathogen ranged from $38.02 \mathrm{~mm}$ ( $A$. sativum) to $76.80 \mathrm{~mm}$ ( $P$. pinnata). However, it was maximum with P.pinnota $(76.80 \mathrm{~mm})$. 
This was followed by $T$. procumbens (76.18mm), L. camera $(76.12 \mathrm{~mm}), \quad P$. longifolia $\quad(76.49 \mathrm{~mm}), \quad Z . \quad$ officinale $(70.88 \mathrm{~mm})$, D. metal $(69.09 \mathrm{~mm})$, A. indica $(66.38 \mathrm{~mm})$ and $O$. sanctum $(51.75 \mathrm{~mm})$. Significantly least radial mycelial growth was recorded with A. sativum $(38.02 \mathrm{~mm})$ over untreated control $(90.00 \mathrm{~mm})$. The mean radial mycelial growth recorded with the plant extracts tested (@10,15 and $20 \%$ each) ranged from $41.27 \mathrm{~mm}$ (A. sativum) to 78.91mm P. longifolia.

However, highest mean radial mycelial growth was recorded with $P$. longifolia $(78.91 \mathrm{~mm})$, and was followed by $P$. pinnata (77.64), T. procumbens $(77.33 \mathrm{~mm})$, L. camera (76.64mm), Z. officinale $(75.47 \mathrm{~mm})$, D. metal $(71.35 \mathrm{~mm})$, A. indica $(67.75 \mathrm{~mm})$, O. sanctum $(61.21 \mathrm{~mm})$ and. Significantly least mean radial mycelial growth was recorded with $A$. sativum $(41.27 \mathrm{~mm})$, over untreated control (90.00mm).

\section{Mycelial growth inhibition}

Result revealed that all the plant extracts tested, significantly inhibited mycelial growth of the tests fungus over untreated control $(00.00 \%)$. Further, it was found that per cent mycelial growth inhibition of the test pathogens was increased with increase in concentrations of the botanicals tested.

At 10 per cent, radial mycelial growth inhibition of the test pathogen ranged from 9.67 (Z. officinale) to $50.99 \%$ (A. sativum). However, significantly highest mycelial growth inhibition was recorded with $A$. sativum $(50.99 \%)$. This was followed by $O$. sanctum (29.22\%), A. indica (23.90\%), D. metal $(18.58 \%)$ and least growth inhibition was recorded, L. camera, $T$. procumbens, $P$. pinnata. P. longifolia, Z. officinale, over untreated control $(00.00 \%)$.
At 15 per cent, radial mycelial growth inhibition of the test pathogen ranged from 11.69 ( $P$. longifolia) to $53.65 \%$ (A. sativum). However, significantly highest mycelial growth inhibition was recorded with $A$. sativum $(53.65 \%)$. This was followed by $O$. sanctum (32.12\%), A. indica (26.00\%), D. metal $(20.32 \%)$ and least growth inhibition was recorded, Z. officinale, L. camera, $T$. procumbens, $P$. pinnata and $P$. longifolia over untreated control $(00.00 \%)$.

At 20 per cent, radial mycelial growth inhibition of the test pathogen ranged from $14.66 \%$ (P.pinnata) to $57.75 \%$ (A. sativum). However, significantly highest mycelial growth inhibition was recorded with $A$. sativum $(57.75 \%)$. This was followed by $O$. sanctum (42.50\%), A. indica (26.24\%), D. metal $(23.23 \%)$ and least growth inhibition was recorded, Z. officinale, L. camera, T. procumbens, $P$. longifolia and $P$. pinnata over untreated control $(00.00 \%)$.

Mean percentage mycelial growth inhibition recorded with all the botanicals tested ranged from $12.31 \%$ ( $P$. longifolia) to $54.13 \%$ ( $A$. sativum). However, A. sativum was found most fungistatic and recorded significantly highest mean mycelial growth inhibition $(54.13 \%)$. The second and third best plant extracts found were $O$. sanctum $(34.61 \%)$ and A. indica $(28.71 \%)$. The remaining all plant extracts found least effective of the test pathogen. Thus, all the plant extracts tested were found fungistatic/ antifungal against Fusarium oxysporum f.sp.cepae.

Thus, on the basis of mean mycelial growth inhibition, the botanicals found most antifungal against Fusarium oxysporum f.sp.cepae. The effectiveness of these botanical extracts may be due to the presence of bioactive and antifungal compounds like phenolic substances, nonvolatile compounds. The presence of ajoene and alliicin in $A$. 
sativum might be the reason for inhibition of Fusarium oxysporum $f$ sp.cepae (Mahadevan, 1982; Yoshida et al., 1987; Naganawa et al., 1996; Sahavaraj et al., 2006).

These results of the present study are in consonance with the finding of several previous workers. Botanical / plant extracts viz., A. sativum, Z. officinale, D. metal, P.pinnata and $A$. indica were reported antifungal / fungistatic against, Fusarium oxysporum $f$. sp.cepae earlier by several workers (Brower and Locky, 2000; Agbenin and Marley, 2006; Taskeen-Un-Nisa et al., 2011; Hadi and Kashefi, 2013; Singh et al., 2014).

Aqueous extracts of 9 botanicals evaluated in vitro $(10 \%, 15 \%$ and $20 \%)$ were found fungistatic/antifungal to A.niger. However, $A$. sativum was found most fungistatic and recorded significantly highest mean mycelial growth inhibition $(53.01 \%)$. The second and third best plant extracts found were $O$. sanctum $(40.37 \%)$ and A. indica $(24.55 \%)$. The remaining all plant extracts found least effective of the test pathogen. Aqueous extracts of 9 botanicals evaluated in vitro $(10 \%, 15 \%$ and $20 \%)$ were found fungistatic/antifungal to Fusarium oxysporum f.sp.cepae. However, A. sativum was found most fungistatic and recorded significantly highest mean mycelial growth inhibition $(54.13 \%)$. The second and third best plant extracts found were $O$. sanctum $(34.61 \%)$ and A. indica $(28.71 \%)$. The remaining all plant extracts found least effective of the test pathogen. Thus, all the plant extracts tested were found fungistatic/ antifungal against Fusarium oxysporum f.sp.cepae.

Thus from the results obtained on various aspects during investigation on post-harvest and storage diseases of onion, following conclusions are being drawn

Aqueous extracts of all the nine botanicals evaluated in vitro found fungistatic/antifungal to both the pathogen (A.niger and Fusarium oxysporum $f$. sp. cepae). However, A.satium, $O$. sanctum and neem oil were effective against both the pathogen.

\section{References}

Agbenin N. Ogechi and Marley P.S., 2006. Studied that in vitro assay of some plant extracts against Fusarium oxysporum f.sp.lycopersici causal agent of tomato wilt. Journal of Plant Protection Research. 46(3): 215-220.

Avasthi Shubhi, Gautam, K.Ajay and Bhadauria Rekha, 2010. Antifungal activity of plant products against Aspergillus niger. A potential application in the control of spoilage fungus. An International Journal 2(1): 53-55.

Bhagchandani, A., Netrapal, P.M., Narendra singh and Choudhuri, B., 1980. White onion for dehydration. Indian Hort. 24: 7-9.

Bohannec B., Jakse M., and Havey M.J., 2003.Genetic analyses of gynogenetic haploid production in onion. J.Am. Soc. Hort. Sci., 128, 571-574.

Brower, J.H. and Locky 2000. Effect of botanical extracts on the population density of Fusarium oxysporum in soil and control of Fusarium wilt in the green house. Plant Disease, 3: 300-305.

Fritsch M. and Friesen N., 2002. Evolution, domestication and taxonomy, in Allium crops Science: Recent Advances (H D Rabinowitch and L Currah, Eds), CABI Publishing, Wallingford, pp. 5-30.

Hadi Mahshid and Kashefi Bahareh, 2013. Study on effect of some medicinal plant extracts on growth and spore germination of Fusarium oxysporum schlecht. In vitro. American- Eurasian J. Agric. \& Environ. Sci., 13 (4):581-588. 
Irkin Reyhan and Korukluoglu Mihriban, 2007. Control of Aspergillus niger with garlic, onion and leek extracts. African J. of Biotech. 6(4): 384-387.

Khan S. Zafar and Nasreen Sahera, 2013. Mycotoxic effect of medicinal plants against Helmimthosporium sativum and Aspergillus niger. Research in Pharmacy, 3(4): 16-20.

Maini, S.B., Diwan, B. and Anand, J.C., 1984. Storage behaviour and drying characteristics of commercial cultivars of onion. J. of Food Science and Technology, 21(6):417

NHRDF 2009. Area and production data, National Horticultural Research and Development Foundation, Available at: www.nhrdf.com. Accessed 14 April 2013

Pantastico, B. and Bantista, O.K. 1976. Postharvest handling of tropical vegetable crops. Hort Sci. 11: 122-124.
Quadri, S.M.H., Srivastava, S.R, Bhonde, S.R., Pandey, U.B. and Bhagchandani, P.M., 1982. Fungicidal bioassay against some important pathogen of onion. Pesticides. 16(11):11-16.

Singh Bhupendra Kumar, Srivastava Seweta, Singh Pramod and shukla D.N. 2014. In vitro evaluation of biocontrol agents and botanicals against Wilt incidence of watermelon, tomato and marigold. $J$. Mycopathol. Res. 52(1): 29-34

Sonawane B.N., sumia Fatima, Kale S.R and Shaikh Yasmeen, 2012. Effect of leaf extracts of some medicinal plant against Aspergillus niger a causal organism of storage diseases of Amla. DAV International Journal of science Vol. 1(2).

Tripathi, P.C. and Lawande, K.E., 2003. Onion storage structure for small and marginal farmers. ICAR News, A Science and Technology Newsletter, 9(4):18- 19.

\section{How to cite this article:}

Futane, A.S., B.P. Dandnaik, P.P. Jadhav and Salunkhe, S.S. 2018. Management of Storage Diseases of Onion by Using Different Botanicals. Int.J.Curr.Microbiol.App.Sci. 7(03): 37083719. doi: https://doi.org/10.20546/ijcmas.2018.703.429 